\title{
Análisis de Sensibilidad Computacional de la Respuesta en Frecuencia de la Impedancia de Torres de Transmisión
}

\author{
Esteban Velilla, Jorge Restrepo y Fernando Villada \\ Facultad de Ingeniería, Departamento de Ingeniería Eléctrica, Universidad de Antioquia, Calle 67 No. 53 - \\ 108, Oficina 19-443, Medellín, Colombia (e-mail: esteban.velilla@udea.edu.co; \\ jorge.restrepo@udea.edu.co; fernando.villada@udea.edu.co)
}

Recibido Ago. 19, 2015; Aceptado Oct. 29, 2015; Versión final Nov. 25, 2015, Publicado Feb. 2016

\begin{abstract}
Resumen
Se determina a través del Modelo Hibrido Electromagnético la respuesta en frecuencia de la impedancia vista desde el conductor de guarda. El modelo considera variaciones en la geometría de dos torres; la puesta a tierra y la resistividad del suelo. La evaluación de transitorios electromagnéticos en líneas de transmisión involucra diferentes elementos siendo las torres uno de los que más podría influenciar la magnitud y forma de onda de los transitorios generados. La respuesta en frecuencia presenta un comportamiento predominantemente inductivo en el rango de frecuencia analizado, razón por la cual fue ajustada a la expresión de impedancia de un circuito serie resistivo-inductivo-capacitivo en términos de transformadas de Laplace, obteniéndose valores de inductancia en el rango de lo reportado en la literatura técnica.
\end{abstract}

Palabras clave: transitorios electromagnéticos; impedancia; torres de transmisión; puesta a tierra; respuesta en frecuencia

\section{Computational Sensitivity Analysis of the Impedance Frequency Response of Power Transmission Towers}

\begin{abstract}
The frequency response of the impedance seen from the top of two towers by the Hybrid Electromagnetic Model is determined. The model considers variations in the geometry of the towers, geometry of grounding system, and soil resistivity. The evaluation of electromagnetic transients in transmission lines involves different components being towers the elements that can further influence the magnitude and wave shape of the generated transient. The frequency response shows a predominantly inductive behavior. For that reason, frequency response is fitted to impedance expressions of a series-inductivecapacitive circuit in terms of Laplace transform, obtaining inductance values in ranges similar to values reported in the literature.
\end{abstract}

Keywords: electromagnetic transients; impedance; transmission tower; grounding systems; frequency response 


\section{INTRODUCCIÓN}

Los transitorios electromagnéticos generados en líneas de transmisión (LT) señalan a la impedancia de la torre como el factor más influyente en su comportamiento, el cual depende de la geometría de la estructura, de los parámetros eléctricos del suelo y del tipo de corriente que se inyecte (Baba, 2001). En la literatura técnica se ha mostrado que una reducción en la impedancia de la torre o en la impedancia de la Puesta a Tierra (PaT), logra disminuir la tasa de salidas de LT debidas a descargas eléctricas atmosféricas (Nucci, 2010; Whitehead, 1993). También se ha mostrado que la velocidad de propagación de las ondas electromagnéticas en las torres de transmisión es menor a la velocidad de la luz (Chisholm, 1983), efecto observado en mediciones experimentales, asociándose esta reducción a la inductancia de la torre, la cual se encontró en un rango entre 30 y $60 \mu \mathrm{H}$ para torres circuito sencillo y doble-circuito de $500 \mathrm{kV}$ (Chisholm, 1985).Adicionalmente, Chisholm y Chow mostraron que un $10 \%$ de cambio en la velocidad de propagación de las ondas electromagnéticas en la torre, es equivalente a una variación del $10 \%$ en la impedancia de la resistencia de la base de la torre (Chisholm, 1983).

Investigaciones tanto teóricas como experimentales han tratado de encontrar expresiones que permitan aproximar la impedancia de las torres. Algunos autores han propuesto obtener la impedancia de la torre en función de la geometría y de la forma de onda ingresada, fundamentando su análisis en modelos verticales y horizontales de geometrías cilíndricas conocida como la ecuación de Jordan (Jordan, 1934) o la de Wagner-Hileman (Wagner, 1959; 1961), y con modelos cónicos como el propuesto por Sargent-Darveniza (Sargent, 1967; 1969). Otros autores, aproximaron la impedancia de la torre por medio de geometrías equivalentes (IEEE, 1997), proponiendo expresiones para obtener el valor de la impedancia de las torres a partir de modelos simples (cilíndricos y cónicos), aplicados a diferentes configuraciones de torres según el nivel de tensión eléctrica.

Estos trabajos se fundamentan en la teoría de campos electromagnéticos y la utilización del método directo, el cual consiste en la medida del voltaje ante la inyección de una onda de corriente determinada tanto en su forma de onda como en su magnitud, inyectada a un modelo a escala 0 al equivalente geométrico de la torre de transmisión, con el fin de obtener el valor de impedancia. A partir de esta metodología se ha aproximando la torre con geometrías cónicas, obteniéndose valores de impedancia entre 144-147 $\Omega$ para torres doble-circuito de $160 \mathrm{~m}$ de altura y $14 \mathrm{~m}$ de radio en la base. Para estas mismas torres utilizando el programa computacional FLASH se han obtenido impedancias entre 194-207న (McDermott, 2010).

Algunos autores han focalizado sus estudios en los efectos inductivos de las torres (Chisholm, 1983; Anderson, 1959), para ello aproximaron la torre mediante modelos equivalentes de geometría cilíndrica, encontrando inductancias de $28.5 \mu \mathrm{H}$ para torres doble-circuito de $345 \mathrm{kV}$ de $45.72 \mathrm{~m}$ de altura y $3.81 \mathrm{~m}$ de diámetro en la base. Por otro lado, otros autores se centraron en los efectos capacitivos de las torres (Caswell, 1858; Kawai, 1964). Caswell realizó mediciones para determinar la capacitancia a tierra de una torre doble-circuito de $138 \mathrm{kV}$ de $38.1 \mathrm{~m}$ de altura y $4.572 \mathrm{~m}$ de diámetro de base, obteniendo mediante cálculos numéricos una capacitancia total a tierra de $1200 \mu \mu \mathrm{F}$, valor que fue contrastado con modelos a escala, con los cuales obtuvo una capacitancia de $1250 \mu \mu \mathrm{Fa}$ una frecuencia de 1000 ciclos.

Es de resaltar que en los estudios de transitorios electromagnéticos, la variación de los parámetros con la frecuencia resulta ser un factor fundamental. Esta dependencia podría influenciar significativamente tanto la impedancia de la torre como la de la puesta a tierra y por ende, afectar los transitorios generados cuando una corriente ingrese al sistema. En este sentido Conti y Visacro compararon los resultados obtenidos de la ecuación de Jordan con respecto a la impedancia impulso de la torre obtenida con el Modelo Híbrido Electromagnético (Hybrid Electromagnetic Model, HEM),obteniéndose una buena aproximación entre ambos modelos con respecto a resultados experimentales (Conti y Visacro, 2006).

Debido a que generalmente las descargas eléctricas atmosféricas ingresan a la torre por el cable de guarda, este trabajo evaluó la respuesta en frecuencia de la impedancia vista desde del cable de guarda en 2 torres de transmisión con geometrías diferentes: una doble-circuito y otra de disposición horizontal típica en 500 $\mathrm{kV}$. Para ello se modeló tanto la geometría de las torres como de las puestas a tierra con el HEM, debido a que éste permite considerar cualquier disposición geométrica de los conductores, los diferentes medios involucrados (aire y tierra), la variación de los parámetros eléctricos con la frecuencia (permitividad y permeabilidad), y los efectos de propagación de las ondas electromagnéticas. Las respuestas en frecuencia de las impedancias fueron obtenidas para diferentes casos, considerando variaciones en la geometría de las torres, puestas a tierra y en la resistividad del terreno; éstas fueron ajustadas a la expresión de impedancia de un circuito serie resistivo-inductivo-capacitivo con la finalidad de determinar los parámetros eléctricos y compararlos con los publicados en la literatura técnica. 


\section{RESPUESTA EN FRECUENCIA DE LA IMPEDANCIA (RFI)}

Un modelo adecuado de la torre de transmisión o de la puesta a tierra debe considerar las características físicas de los electrodos (geometría y radio); los parámetros eléctricos del medio (conductividad, permitividad, permeabilidad magnética), su dependencia con la frecuencia; y la propagación de las ondas electromagnéticas (Visacro, 2013). Uno de los modelos que logra involucrar estas tres características es el HEM (Visacro, 1992), el cual evalúa el comportamiento electromagnético de un electrodo de cualquier geometría inmerso en un medio determinado, recurriendo a la segmentación analítica de los electrodos y a la teoría de las imágenes para establecer los diferentes acoples electromagnéticos. En el modelo cada segmento es asumido como una fuente constante de corriente longitudinal y transversal, las cuales generan los efectos conductivos y capacitivos a través de la expresión 1, y los efectos inductivos con la expresión 2.

$$
\begin{aligned}
& V_{i j}=\frac{I_{T_{j}}}{4 \pi(\sigma+j \omega \varepsilon)} \cdot \frac{1}{L_{i} L_{j}} \cdot \int_{0}^{L_{i}} \int_{0}^{L_{j}} \frac{e^{-k \cdot r}}{r} d l_{j} d l_{i} \\
& \Delta V_{i j}=-j \frac{\omega \mu}{4 \pi} \cdot \int_{0}^{L_{i}} \int_{0}^{L_{j}} \frac{\vec{I}_{L_{j}} e^{-k \cdot r}}{r} d l_{j} \overrightarrow{d l_{i}}
\end{aligned}
$$

Siendo $V_{i j}$ el potencial eléctrico generado en el segmento $i$ cuando circula una corriente transversal en el segmento $j\left(I_{T_{j}}\right) ; \Delta V_{i j}$ la diferencia de potencial generada o caída de tensión en el segmento $i$ cuando circula una corriente longitudinal en el segmento $j\left(\vec{I}_{L_{j}}\right) ; \sigma$ la conductividad eléctrica y $\varepsilon$ la permitividad del medio en el cual se encuentra el segmento $j$, parámetros que varían con la frecuencia; $L_{i}$ y $L_{j}$ las longitudes de los respectivos segmentos $i$ y $j$; $r$ la distancia geométrica entre los diferenciales de ambos segmentos y $k$ la constante de propagación del medio.

A partir de las expresiones anteriores y de las relaciones de contorno establecidas por las leyes de Kirchoff en los nodos que unen los segmentos, se genera el sistema lineal a resolver de la forma $A \cdot V=I$, donde I es el vector de corriente que tendrá un valor en el nodo donde se realiza la inyección de corriente y cero en los demás elementos (Gómez, 2006). De esta manera, el HEM realiza el análisis electromagnético variando la frecuencia de la excitación sinusoidal considerando la dependencia de los parámetros eléctricos del medio con la frecuencia, con el fin de obtener el comportamiento de cada una de las variables involucradas en el dominio de la frecuencia, para luego hacer uso de la transformada y transformada inversa de Fourier y así obtener el comportamiento transitorio o dinámico ante inyecciones de señales de corriente en el dominio del tiempo.

De manera general, la respuesta en frecuencia de la impedancia (RFI) es la relación obtenida de la tensión generada en un lugar definido cuando una corriente sinusoidal con una frecuencia específica ingresa al sistema. Esto es, la relación entre la señal de salida (tensión) y la señal de entrada en el sistema (corriente), relación que es evaluada en el caso del HEM en el rango de frecuencia que va desde aproximadamente cero hasta un $\mathrm{MHz}$.

La respuesta en frecuencia generalmente es expresada en variables complejas, y por tal razón, el uso de diagramas de magnitud y fase es convencionalmente utilizado para representar dicha relación. A través de estos diagramas se han identificado problemas mecánicos en transformadores (Aponte, 2011), o en otros casos, a partir de la respuesta en frecuencia se han obtenido modelos matemáticos que han permitido reproducir tanto la respuesta en frecuencia como la respuesta dinámica de un sistema ante diversas excitaciones (Velilla, 2006).

\section{ANÁLISIS DE SENSIBILIDAD DE LA RFI}

Con la finalidad de observar el efecto de algunas variables en la RFI vista desde el cable de guarda, se modelaron con el HEM 2 torres de transmisión: una con disposición geométrica de los conductores de forma Horizontal $(\mathrm{H})$ y otra con disposición vertical de los conductores, Doble Circuito (DC), ambas geometrías simétricas con respecto al eje vertical. Las torres fueron construidas por etapas con el objeto de ir considerando cada vez más elementos conductores que representen la geometría de las torres en evaluación. Adicionalmente, se consideró cambios en la altura de la torre y se varió la resistividad del terreno de 2 geometrías de PaT.

\section{Respuesta en frecuencia de la impedancia por etapas}

La construcción por etapas de ambas torres se realizó considerando una altura total entre el suelo y el cable de guarda de aproximadamente $31.25 \mathrm{~m}$ y un radio de la base de la torre de $7.164 \mathrm{~m}$ para la $\mathrm{H}$ y de $10.8 \mathrm{~m}$ 
para la DC; las torres son construidas por etapas partiendo de un conductor vertical simple y uno horizontal que representa los conductores de guarda ( $\mathrm{H} 1$ y DC1), luego considerando la base de las torres $(\mathrm{H} 2$ y DC2), y en la última etapa, considerando más elementos conductores con la finalidad de llevar en cuenta el efecto de los brazos y representar mejor la geometría de la torre (H3 y DC3), Fig. 1 y Fig. 2. Todos los electrodos son considerados como conductores cilíndricos de radio de $10 \mathrm{~mm}$; adicionalmente, se consideró el suelo con resistividad de $100 \Omega \cdot \mathrm{m}$, y no se consideró ninguna PaT con el fin de solo observar el efecto de la torre.

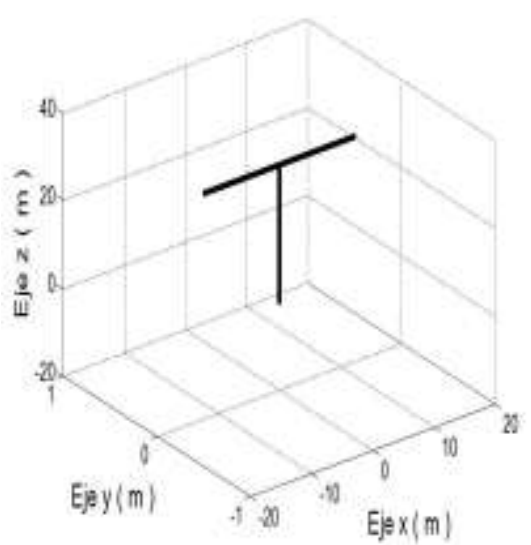

a) Etapa $\mathrm{H} 1$

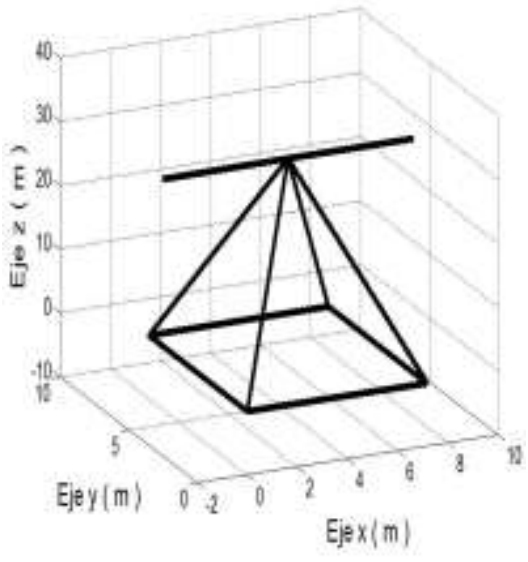

b) Etapa $\mathrm{H} 2$

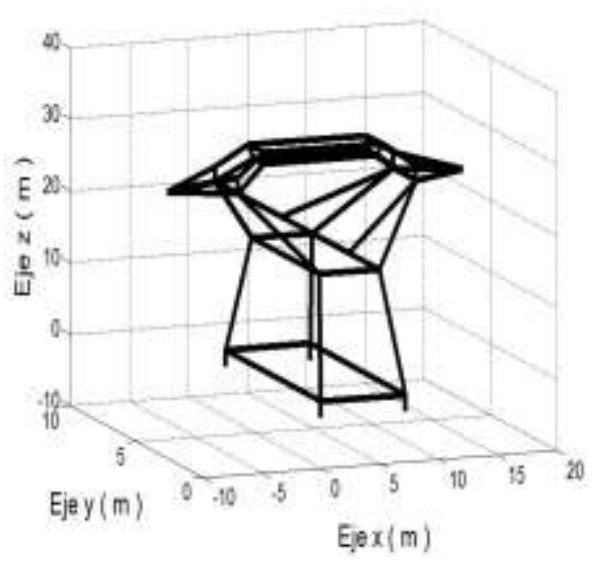

c) Etapa H3

Fig. 1. Construcción por etapas de la torre Horizontal $(H)$

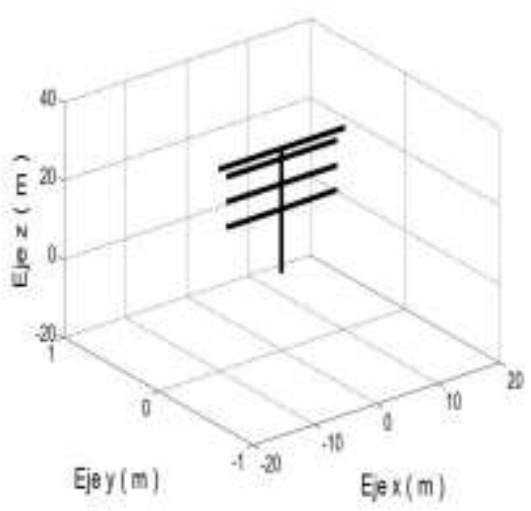

a) Etapa DC1

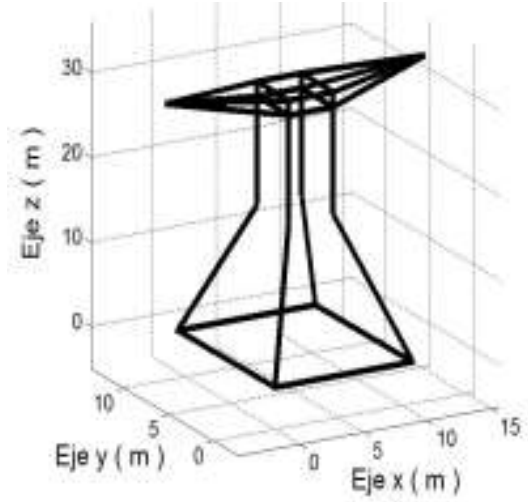

b) Etapa DC2

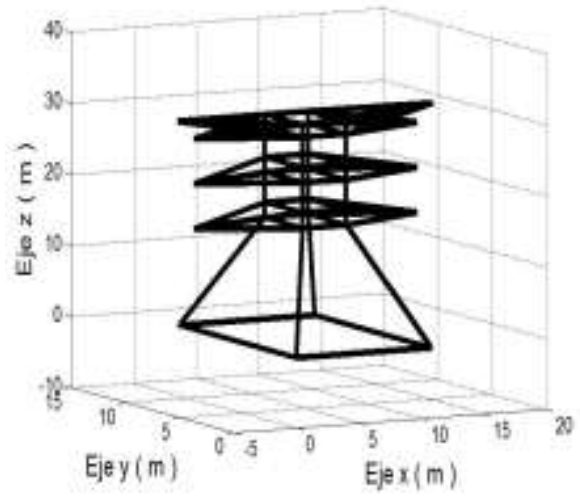

c) Etapa DC3

Fig. 2. Construcción por etapas de la torre Doble Circuito (DC)

En la Fig. 3 se presentan las RFI de ambas torres por etapas, observándose en todos los casos el efecto inductivo reflejado en el ángulo de la impedancia, el cual tiene a $90^{\circ}$ en la medida que la frecuencia tiende a $1 \mathrm{MHz}$. De igual manera se observa una variación significativa en la magnitud de la impedancia a bajas frecuencias en la medida que se consideran más elementos, obteniéndose la mayor magnitud con la geometría simple y el menor valor con la geometría completa. Es de resaltar que la RFI de la torre $\mathrm{H}$ presenta valores de impedancia menores a los obtenidos para la torre DC en todo el rango de frecuencia, esto es, la impedancia vista desde el cable de guarda en una torre $\mathrm{H}$ es menor a la observada en una torre DC.

\section{Variación de la resistividad del terreno considerando 2 PaT diferentes}

En estudios previos (Velilla, 2013), se analizaron diversas geometrías de PaT en torres de transmisión, encontrándose que las 2 geometrías presentadas en la Fig. 4 presentan bondades debido a la reducción de la impedancia en el rango de frecuencia analizado y por ende una reducción en las tensiones originadas para diferentes formas de onda de excitación en un amplio rango de resistividades (hasta algunos $\mathrm{k} \Omega \cdot \mathrm{m}$ ). 


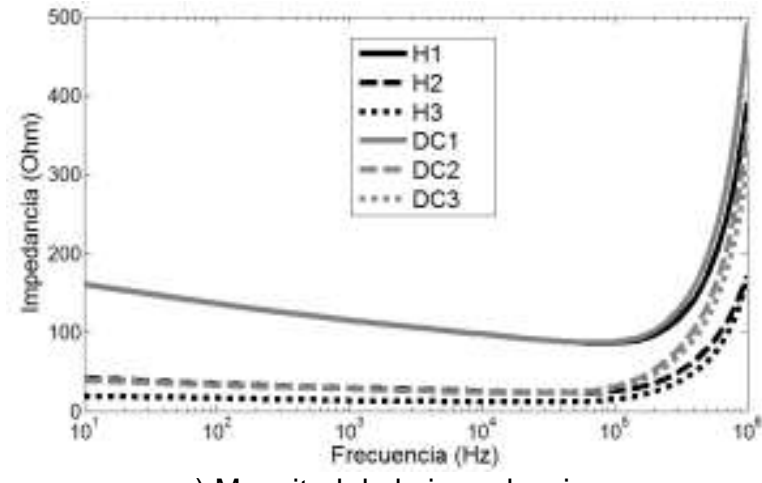

a) Magnitud de la impedancia

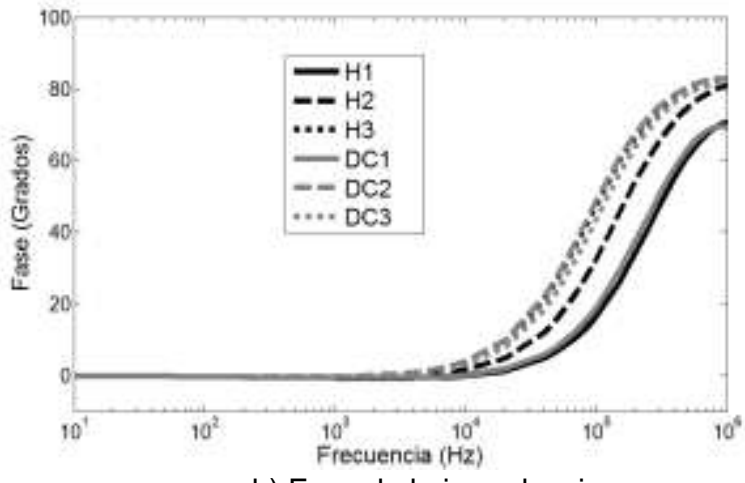

b) Fase de la impedancia

Fig. 3. Respuesta en frecuencia de la impedancia de las torres $\mathrm{DC}$ y $\mathrm{H}$

Por esta razón, para este análisis se consideraron las torres de la etapa 3 y las geometrías de PaT de la Fig. 4 enterradas a $0.6 \mathrm{~m}$, con varillas de $2.4 \mathrm{~m}$, contrapesos de $14.1 \mathrm{~m}$ de longitud y una malla interna de iguales dimensiones a la base de la torre. Adicionalmente, se varió la resistividad del terreno en 100, 500 y $1000 \Omega \cdot \mathrm{m}$. En la Fig. 5 y Fig. 6 se presentan los resultados obtenidos, observándose que considerar la PaT trae un efecto positivo en la reducción de la impedancia principalmente en baja frecuencia, rango en el cual la influencia de la resistividad se hace muy visible. De igual manera se observa que la fase de la impedancia varia con la resistividad, y en la medida que se aumenta la resistividad del suelo, la fase de la impedancia presenta un desplazamiento hacia la derecha, esto es, se aumentan los efectos capacitivos en la impedancia cuando se considera el conjunto Torre-PaT.

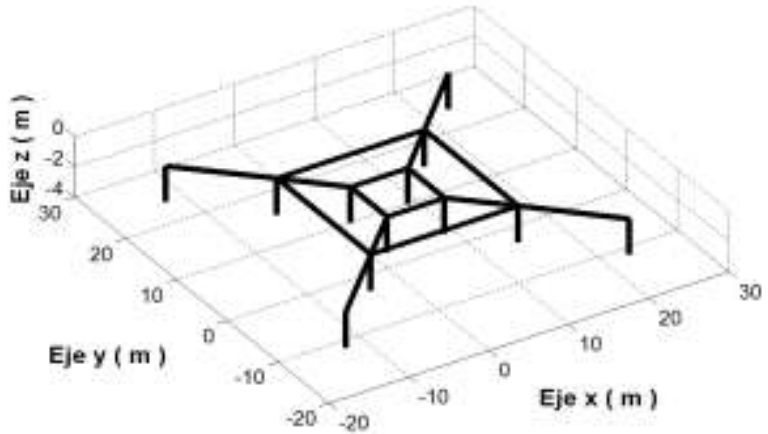

a) PaT1

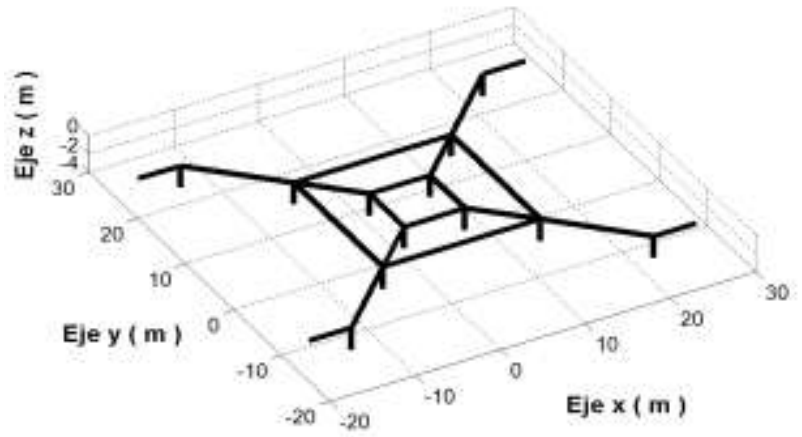

b) $\mathrm{PaT2}$

Fig. 4. Configuraciones de PaT

Resultados similares fueron reportados en torres DC de $245 \mathrm{kV}$ de $45-50 \mathrm{~m}$ de altura y bases de $6.86 \times 8.86$ m a 10×10 m, para diferentes valores de resistividad (Harid, 2006). Resaltándose que a bajas frecuencias el voltaje de la torre es constante y proporcional a la resistividad del suelo; que la impedancia de la torre crece gradualmente con la frecuencia; que a frecuencias de $1 \mathrm{MHz}$ la magnitud de la impedancia llega a ser menos dependiente de la resistividad del suelo; y que en la medida que se aumenta la frecuencia, los efectos inductivos llegan a tener un efecto predominante.

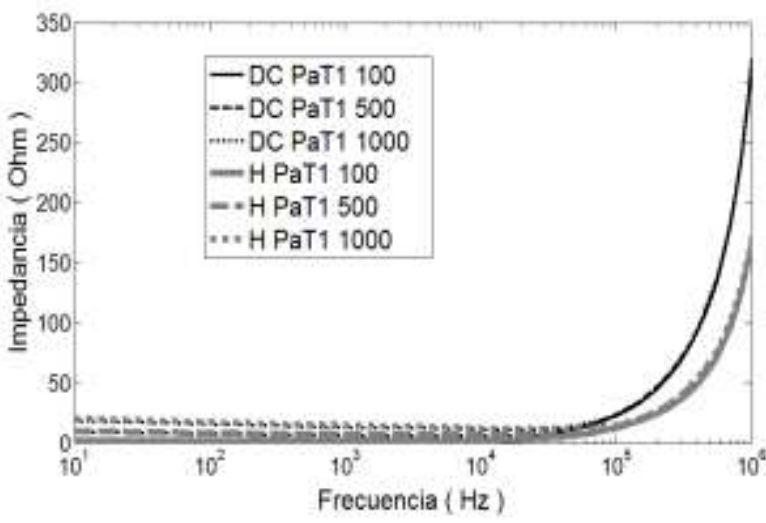

a) Magnitud de la impedancia

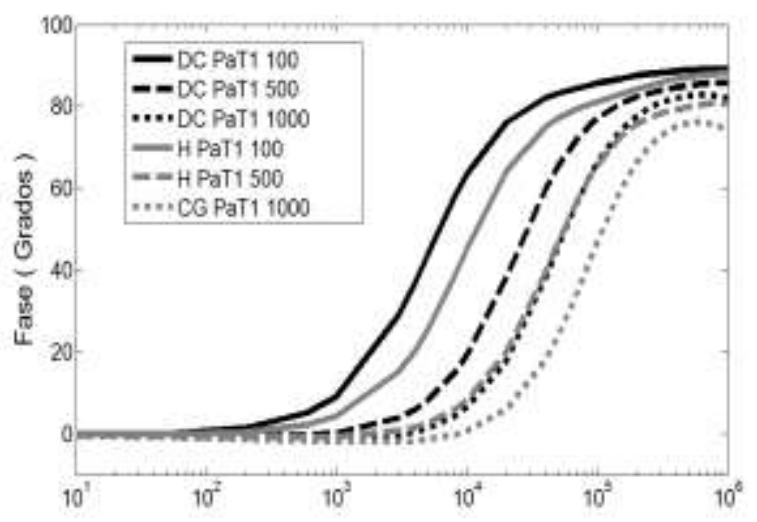

b) Fase de la impedancia

Fig. 5. RF del conjunto torre- PaT1 


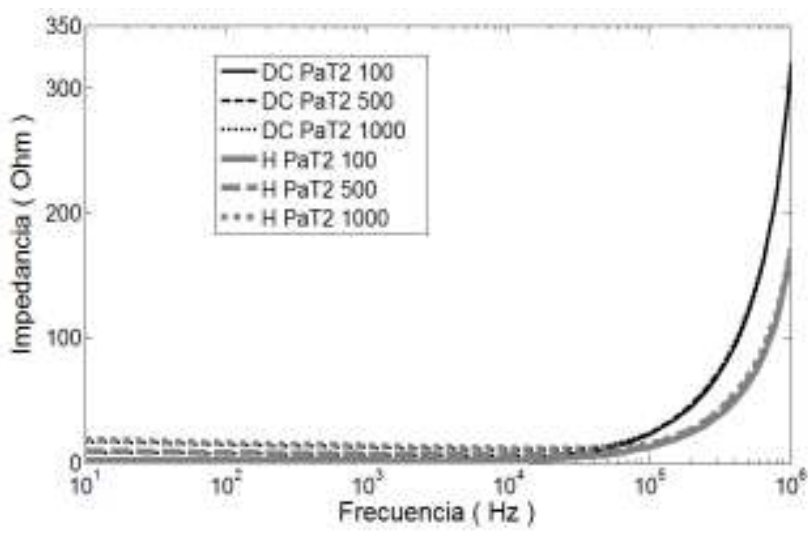

a) Magnitud de la impedancia

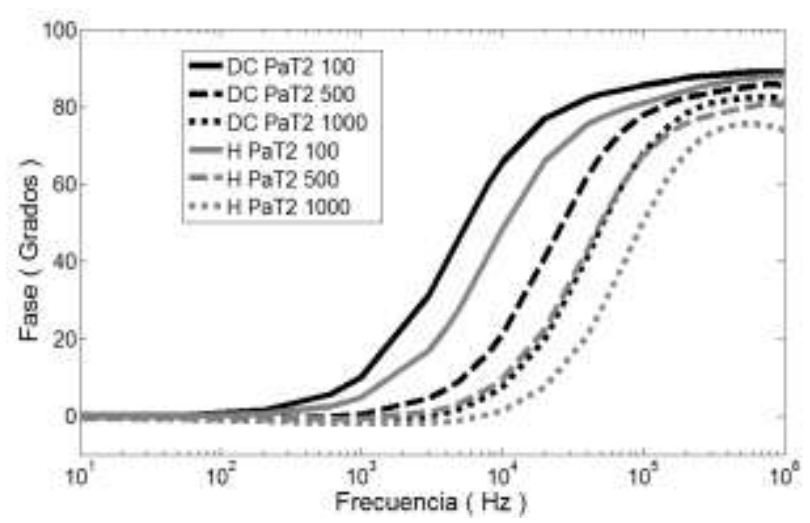

b) Fase de la impedancia

Fig. 6. RF del conjunto torre - PaT2

\section{Variación en la altura de la torre}

El análisis consistió en considerar la PaT2 y las torres de la etapa 3 que representan mejor la geometría de las torres y variar la altura en 15, 20,31 y $47 \mathrm{~m}$ respectivamente, con las mismas consideraciones del caso anterior. Los resultados obtenidos son presentados en la Fig. 7, observándose que un aumento en la altura de las torres, se refleja en un aumento de la magnitud de la impedancia en las frecuencias altas, y en un desplazamiento hacia la izquierda de la fase de la impedancia con la frecuencia, indicando esto un comportamiento más inductivo con el aumento de altura.

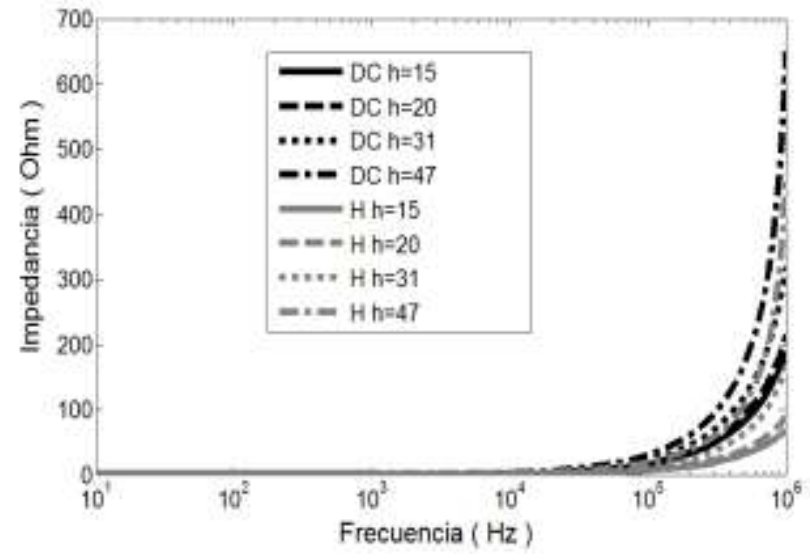

a) Magnitud de la impedancia

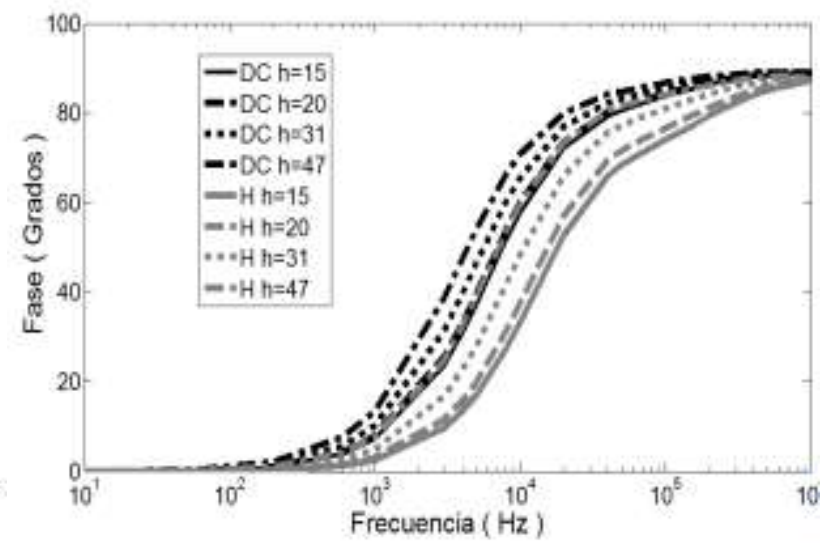

b) Fase de la impedancia

Fig. 7. RF de la torre $\mathrm{DC}$ y $\mathrm{H}$ variando la altura

\section{AJUSTES DE LA RFI A CIRCUITOS RLC SERIE}

Las RFI obtenidas presentaron un comportamiento muy similar al observado en la respuesta en frecuencia de la ganancia de tensión en transformadores (Velilla, 2012), comportamiento que se caracterizó por una tendencia aproximadamente constante entre 0 y $10^{5} \mathrm{~Hz}$; un aumento considerablemente de la ganancia de tensión después de los $10^{5} \mathrm{~Hz}$ y un cambio en la concavidad de la fase de ésta. En el caso de las torres, el cambio en la concavidad de la fase de la impedancia se hizo más evidente con el aumento de la altura de las torres y con la variación en la resistividad del suelo, de igual manera, la RFI de la torre $\mathrm{H}$ siempre presentó un comportamiento más homogéneo en el rango de frecuencia analizado y de menor magnitud al obtenido para la torre DC.

En vista de que a partir de las RFI se han obtenido modelos matemáticos que reproducen el comportamiento observado en el dominio de la frecuencia, en este trabajo se optó por realizar el ajuste de la RFI a modelos circuitales o expresiones matemáticas que representan un circuito eléctrico determinado. Para ello se tuvo en cuenta que las RFI de las torres no fueron constantes en todo el rango de frecuencia analizado y por tanto no podrían ser representadas adecuadamente por una resistencia; que las RFI presentaron un crecimiento significativo con la frecuencia, comportamiento que podría ser explicado por la componente inductiva presente en la impedancia de la torre, tema que ha sido abordado ampliamente en la literatura; y que las RFI presentaron un cambio de concavidad muy notorio en la fase de la impedancia, el cual podría ser explicado por la interacción entre efectos inductivos y capacitos. 
Un modelo circuital que involucra los componentes anteriores es el circuito serie conformado por una resistencia, inductancia y capacitancia, el cual tiene asociada una impedancia en el dominio de la frecuencia en términos de Laplace dada por la expresión 3.

$Z(s)=\frac{V(s)}{I(s)}=R+L \cdot s+\frac{1}{C \cdot s}$

Siendo $R$ la resistencia, $L$ la inductancia, $C$ la capacitancia y $s$ el término complejo de la transformada de Laplace dado por $\mathrm{s}=-2 \pi f \cdot j$, donde $\mathrm{f}$ es la frecuencia en $\mathrm{Hz}$ y $\mathrm{j}$ representa el número complejo.

Con la finalidad de encontrar los parámetros $R, L$ y $C$ de la impedancia $Z(s)$ y reproducir adecuadamente las $\mathrm{RFI}$ de las torres, se definió como función objetivo la minimización del error entre la RFI obtenida con el HEM y la evaluación de la expresión de impedancia con los 3 parámetros en cada una de las frecuencias analizadas, expresión 4. Este problema de optimización se resolvió con el método Simplex (Velilla, 2008).

Error $=\sqrt{\sum_{i=1}^{n}\left(R F I_{f(i)}-Z(R, L, C)_{f(i)}\right)^{2}}$

Donde $f$ es el vector de frecuencias con las cuales se evaluó la RFI, $n$ es el número total de frecuencias presentes en el vector $f, R F I_{f(i)}$ es el valor de la RFI obtenida con el HEM para la frecuencia $f(i)$ y $Z(R, L, C)_{f(i)}$ es la evaluación de la impedancia en la frecuencia $f(i)$.

En las Tablas 1, 2, 3 y 4 se presenta los parámetros de la impedancia obtenidos al ajustar las RFI al modelo circuital para los análisis considerados. Adicionalmente, con la finalidad de brindar información sobre el comportamiento de la impedancia en el dominio del tiempo, esto es, comportamiento transitorio, se incluyo la impedancia impulso definida como la máxima tensión generada cuando la corriente de excitación es una onda $1.2 / 50 \mu \mathrm{s}\left(Z=V_{P} / I_{P}\right)$.

Tabla 1. Parámetros circuitales al variar los elementos de las torres

\begin{tabular}{|c|c|c|c|c|c|c|}
\hline Circuito & $\mathrm{H} 1$ & $\mathrm{H} 2$ & $\mathrm{H} 3$ & $\mathrm{DC} 1$ & $\mathrm{DC} 2$ & $\mathrm{DC} 3$ \\
\hline $\mathrm{Z}_{\text {imp }}(\Omega)$ & 125.43 & 51.67 & 40.96 & 141.03 & 87.08 & 76.52 \\
\hline $\mathrm{R}(\Omega)$ & 99.29 & 24.79 & 12.85 & 105.76 & 26.64 & 27.62 \\
\hline $\mathrm{L}(\mu \mathrm{H})$ & 53.19 & 25.56 & 23.00 & 63.82 & 49.37 & 42.27 \\
\hline $\mathrm{C}(\mathrm{mF})$ & 20.08 & 77.05 & 156.9 & 19.91 & 76.57 & 74.85 \\
\hline
\end{tabular}

En la Tabla 2 y Tabla 3 se presentan los resultados de los ajustes de las RFI al modelo circuital considerando las 2 PaT en las torres, observándose que en la medida que se aumenta la resistividad del terreno, tanto la impedancia impulso como el valor de resistencia encontrado aumentan. De igual manera se observa que los valores de inductancia encontrados no presentan una variación significativa ante los cambios de resistividad, mientras que la capacitancia si presenta variaciones considerables.

En la Tabla 4 se presentan los resultados de los ajustes obtenidos al variar las alturas en las torres. Los resultados indican que al aumentar la altura, tanto la inductancia como la impedancia impulso aumentan. Es de resaltar que las $\mathrm{RFI}$ de la torre $\mathrm{H}$, presentaron una respuesta más plana y magnitudes de impedancia menores que las obtenidas con las RFI de la torre DC. Este comportamiento se observa en los valores numéricos de los parámetros obtenidos con el ajuste propuesto, valores que siempre fueron mejores en para la torre $\mathrm{H}$.

Tabla 2: Parámetros circuitales obtenidos al variar la resistividad del terreno en la torre $\mathrm{H}$

\begin{tabular}{|c|c|c|c|c|c|c|c|c|}
\hline \multirow{2}{*}{$\begin{array}{c}\text { Resistividad } \\
(\Omega \cdot \mathrm{m})\end{array}$} & \multicolumn{4}{|c|}{ PaT1 } & \multicolumn{4}{c|}{ PaT2 } \\
\cline { 2 - 10 } & $\mathrm{Z}_{\text {imp }}(\Omega)$ & $\mathrm{R}(\Omega)$ & $\mathrm{L}(\mu \mathrm{H})$ & $\mathrm{C}(\mathrm{mF})$ & $\mathrm{Z}_{\text {imp }}(\Omega)$ & $\mathrm{R}(\Omega)$ & $\mathrm{L}(\mu \mathrm{H})$ & $\mathrm{C}(\mathrm{mF})$ \\
\hline 100 & 38.07 & 2.70 & 23.20 & 1126.40 & 38.06 & 2.60 & 23.20 & 1230.7 \\
\hline 500 & 39.82 & 10.81 & 23.38 & 144.25 & 39.77 & 10.59 & 23.38 & 157.78 \\
\hline 1000 & 41.59 & 18.43 & 23.62 & 53.25 & 41.48 & 18.10 & 23.59 & 58.19 \\
\hline
\end{tabular}


Tabla 3: Parámetros circuitales obtenidos al variar la resistividad del terreno en la torre DC

\begin{tabular}{|c|c|c|c|c|c|c|c|c|}
\hline \multirow{2}{*}{$\begin{array}{c}\text { Resistividad } \\
(\Omega \cdot \mathrm{m})\end{array}$} & \multicolumn{4}{|c|}{ PaT1 } & \multicolumn{4}{c|}{ PaT2 } \\
\cline { 2 - 9 } & $\mathrm{Z}_{\text {imp }}(\Omega)$ & $\mathrm{R}(\Omega)$ & $\mathrm{L}(\mu \mathrm{H})$ & $\mathrm{C}(\mathrm{mF})$ & $\mathrm{Z}_{\text {imp }}(\Omega)$ & $\mathrm{R}(\Omega)$ & $\mathrm{L}(\mu \mathrm{H})$ & $\mathrm{C}(\mathrm{mF})$ \\
\hline 100 & 71.44 & 2.28 & 44.02 & 820.7 & 71.40 & 2.22 & 43.99 & 869.8 \\
\hline 500 & 72.40 & 9.58 & 43.85 & 150.8 & 72.36 & 9.41 & 43.85 & 162.4 \\
\hline 1000 & 73.25 & 17.00 & 43.75 & 57.97 & 73.17 & 16.73 & 43.73 & 62.56 \\
\hline
\end{tabular}

Tabla 4: Parámetros circuitales obtenidos al variar la altura de la torre

\begin{tabular}{|c|c|c|c|c|c|c|c|c|}
\hline \multirow{2}{*}{$\begin{array}{c}\text { Altura } \\
(\mathrm{m})\end{array}$} & \multicolumn{4}{|c|}{$\mathrm{H}$} & \multicolumn{4}{c|}{$\mathrm{DC}$} \\
\cline { 2 - 9 } & $\operatorname{Zimp}(\Omega)$ & $\mathrm{R}(\Omega)$ & $\mathrm{L}(\mu \mathrm{H})$ & $\mathrm{C}(\mathrm{mF})$ & $\operatorname{Zimp}(\Omega)$ & $\mathrm{R}(\Omega)$ & $\mathrm{L}(\mu \mathrm{H})$ & $\mathrm{C}(\mathrm{mF})$ \\
\hline 15 & 18.12 & 2.20 & 10.5 & 2114 & 46.63 & 1.90 & 28.00 & 1558.60 \\
\hline 20 & 22.64 & 2.30 & 13.3 & 1908 & 52.22 & 2.00 & 31.50 & 1373.80 \\
\hline 31 & 38.06 & 2.60 & 23.2 & 1230 & 71.40 & 2.22 & 43.99 & 869.78 \\
\hline 47 & 78.92 & 4.13 & 49.96 & 437.3 & 117.68 & 3.43 & 74.39 & 350.63 \\
\hline
\end{tabular}

\section{CONCLUSIONES}

Se realizó un análisis de sensibilidad de la respuesta en frecuencia de la impedancia vista desde el cable de guarda en 2 torres de transmisión: una con disposición horizontal de los conductores $(\mathrm{H})$ y otra con doble disposición vertical de los conductores (doble-circuito). Para ello se varió tanto la geometría de las torres y la altura de éstas, como la geometría de las puestas a tierra y la resistividad del terreno. Observándose en todos los casos que la torre con geometría horizontal $(\mathrm{H})$, presenta una RFI más baja en todo el rango de frecuencia analizado, una menor impedancia impulso y un comportamiento menos inductivo comparado con el comportamiento obtenido para la torre que tiene una disposición vertical de los conductores (DC).

El comportamiento inductivo se evidenció en el desplazamiento de la fase de la impedancia hacia la izquierda en la medida que se aumenta la altura de la torre o se disminuye la resistividad del terreno. De igual manera, este comportamiento predominantemente inductivo se observó en los resultados obtenidos del ajuste de las RFI al circuito serie resistivo-inductivo-capacitivo, a través del cual se encontraron valores de inductancia que están en el rango de lo reportado en la literatura técnica para torres con dimensiones y geometrías similares.

Por último, en los casos analizados se observó que la geometría de la torre, la puesta a tierra y la resistividad del terreno juegan un papel importante en la RFI que vería una descarga eléctrica atmosférica desde el cable de guarda. Encontrándose que las torres $\mathrm{H}$ presentarán menores sobretensiones comparadas con las torres DC para diferentes formas de onda debido al comportamiento más plano de la RFI y a los bajos valores de impedancia impulso. De esta manera, a través de la respuesta en frecuencia de la impedancia se podrán valorar los transitorios electromagnéticos en líneas de transmisión y equipos asociados, permitiendo así tomar decisiones para reducir las sobretensiones generadas y de esta manera disminuir la tasa de salidas por descargas atmosféricas.

\section{AGRADECIMIENTOS}

Los autores agradecen a la Universidad de Antioquia (UdeA) por el apoyo del proyecto "Sostenibilidad 20142015" para el desarrollo de este trabajo.

\section{REFERENCIAS}

Aponte G., Herrera W., González C., Pleite J., Implementación de un Modelo de un Transformador Eléctrico para el Análisis de su Respuesta en Frecuencia, Información Tecnológica, 22(4), 59-72 (2011)

Anderson, J.G. and J.H. Hagenguth, Magnetic Fields Around a Transmission Line Tower, AIEE Transactions on Power Apparatus and Systems, 78, 1644-1650 (1959)

Baba Y., Ishii M., Numerical Electromagnetic Field Analysis of Lightning Current in Tall Structures, IEEE Transactions on Power Delivery, 16(2), 324-328 (2001) 
Caswell R. W., Johnson I. B., Koncel E. F., and Schultz N. R., Lightning Performance of 138-kV Twin-Circuit Transmission Lines of Commonwealth Edison Company-Operating Experience and Field Studies, IEEE Trans., 77(3), 1480-1491 (1958)

Chisholm W., Chow Y.I., Lightning Surge Response of Transmission Towers, IEEE Transactions on Power Apparatus and Systems, 102(9), 3232-3242 (1983)

Chisholm W., Chow Y.I., Travel Time of Transmission Towers, IEEE Transactions on Power Apparatus and Systems, 104(10), 2922-2928 (1985)

Conti A., Visacro S., Revision, Extension, and Validation of Jordan's Formula to Calculate the Surge Impedance of Vertical Conductors, IEEE Transactions on Electromagnetic Compatibility, 48(3), 530-536 (2006)

Gómez H., Velilla E., Moreno G., Valencia J., Respuesta Transitoria de las puestas a tierra en la protección contra rayos. Software GTIERRAS. Revista Facultad de Ingeniería, 36, 21-32 (2006)

Harid N., H. Griffiths, and A. Haddad, A New Frequency Dependent Surge Impedance Calculation Method for High-Voltage Towers, IEEE Transactions on Power Delivery, 21(3), 1430-1437 (2006)

IEEE Std. 1243, Design Guide for Improving the Lightning Performance of Transmission Lines (1997)

Jordan C. A., Lightning Computation for Transmission Lines with Overhead Ground Wires. General Electric Rev., 37, 130-137 (1934)

Kawai M., Studies of the Surge Response on a Transmission Line Tower, IEEE Trans., 83, 30-34 (1964)

McDermott, T.E., A New version of the IEEE Flash program, Proposed Transmission and Distribution Conference and Exposition, 2010 IEEE PES, 19-22 (2010)

Nucci C.A., A Survey on CIGRE And IEEE Procedures for the Estimation of The Lightning Performance of Overhead Transmission and Distribution Lines, Asia-Pacific International Symposium on Electromagnetic Compatibility, April 12 - 16, Beijing, China. ISBN: 978-1-4244-5621-5 (2010)

Sargent M. and Darveniza, M, The calculation of the double circuit outage rates of transmission lines, IEEE Trans. Power Apparatus and Systems, 86, 665-678 (1967)

Sargent M, Darveniza M, Tower Surge Impedance, IEEE Transactions on Power Apparatus and Systems, 88(5), 680-687 (1969)

Velilla, E., Moreno G., Programa Computacional para la Toma de Decisiones de Geometría de Puesta a Tierra en Torres de Transmisión, Vicerrectoría de Investigación, Universidad de Antioquia, Medellín, Colombia (2013)

Velilla E., Muñoz J., Ramírez E., Valencia J., Transferencia de Impulsos y Ganancia de Tensión en Transformadores Monofásicos de Baja Potencia, Información Tecnológica, 23(1), 79-88 (2012)

Velilla, E., Valencia, J.A. , Moreno, G., Using genetic algorithm and the Simplex method to obtain equivalent circuits of the grounding systems, Transmission and Distribution Conference and Exposition: Latin America, 2008 IEEE/PES, 1- 5, Bogotá, Colombia 13 al 15 de Agosto (2008)

Velilla, E., Valencia, J.A., Vanegas, A., Moreno, G., Some Methodologies for Designing Circuits that Approximate the Frequency Response of a Grounding System, International Conference on Grounding and Earthing \& $2^{\text {nd }}$ International Conference on Lightning Physics and Effects, Maceió, Brazil 26 al 29 de Noviember (2006)

Visacro S.; Guimarães, M.; Araujo L.; Experimental impulse response of grounding grids. Electric Power Systems Research 94, 92-98 (2013)

Visacro, S. Modelagem de Aterramentos Elétricos. PhD Thesis, Universidade Federal do Rio de Janeiro (1992)

Wagner, C. F.; Hileman, A. R., Surge Impedance and Its Application to the Lightning Stroke, Power Apparatus and Systems, Part III, Transactions of the American Institute of Electrical Engineers, 80(33), 1011-1020 (1961) 
Wagner, C. F.; Hileman, A. R., A New Approach to Calculation of Lightning Performance of Transmission Lines (part II), AIEE Trans., pt. III, 78, 996-1021 (1959)

Wagner, C. F.; Hileman, A. R., A New Approach to Calculation of Lightning Performance of Transmission Lines (part III), AIEE Trans., pt. III, 79, 589-603 (1960)

Withehead J., Chisholm W., IEEE Working Group Report Estimating Lightning Performance of Transmission Lines II-Update to Analytical Models, IEEE Transactions on Power Delivery, 8(3), 1254-1268 (1993) 